Original Research Paper

\title{
Effect of Ultra-High Pressure Technology on Isomerization and Antioxidant Activity of Lycopene in Solanum Lycopersicum
}

\author{
${ }^{1}$ Siqun Jing, ${ }^{2}$ Zainixi Lasheng, ${ }^{2}$ Saisai Wang and ${ }^{2}$ Hui Shi \\ ${ }^{1}$ Yingdong Food College, Shaoguan University, Daxue Road 288, Zhenjiang District, Shaoguan, Guangdong 512005, China \\ ${ }^{2}$ College of Life Sciences and Technology, Xinjiang University, Shengli Road 14, Urumqi, Xinjiang 830046, China
}

\author{
Article history \\ Received: 04-03-2019 \\ Revised: 20-05-2020 \\ Accepted: 19-06-2020 \\ Corresponding Author: \\ Siqun Jing \\ Yingdong Food College, \\ Shaoguan University, Daxue \\ Road 288, Zhenjiang District, \\ Shaoguan, Guangdong 512005, \\ China \\ Email: neverbeshy2012@163.com
}

\section{Introduction}

Lycopene, a functional natural red pigment, is rich in fruits and vegetables like GAC, tomatoes, watermelon, pink guava and red bell pepper and widely used in healthy food, pharmaceuticals and cosmetics due to its antioxidant properties. The lycopene in tomato is considered to possess the highest antioxidant activity. It is well known that $90-98 \%$ of lycopene from a natural source existed in the form of trans. A number of studies have suggested that cis-isomers of lycopene could be better absorbed in the human body than the all-transisomer due to the change in solubility and physical properties of lycopene by the cis-isomerization. According to researches, lycopene could inhibit the proliferation of breast cancer cells (Murakami et al., 2017), decrease the risk of heart disease (Li et al., 2017) and inflammation (Peng et al., 2017) induced by rhinovirus infection and lipopolysaccharide
(Saedisomelia et al., 2009; Armoza et al., 2012). They also show that lycopene have protective effect on serum cholesterol and blood pressure (Petyaev et al., 2014). Therefore, more and more scientists are devoted to the study of lycopene isomerization. Phan-Thi et al. (2014) observed the impact of heating on cis-isomerization of oil-free lycopene in hexane at 50 and $80^{\circ} \mathrm{C}$ during 240 $\mathrm{min}$ and found the initial all-trans-form isomerized to the 13 -cis isomer more rapidly at $80^{\circ} \mathrm{C}$. Cooperstone et al. (2016) found the formation of cis-lycopene in intense thermal pasteurization processing, but high pressure sterilization limited the overall lycopene isomerization compared to the equivalent thermal sterilization process. Takehara et al. (2015) reported that (15Z) lycopene was prepared by thermal isomerization of (all-E)-lycopene derived from tomatoes. Heat treament is widely used in food processing. Heat treatment to tomato productions can make trans-lycopene transform into cis-struture while some other active substances in lycopene, such as VE and 
phytosterol, were also oxidized and degraded (Sy et al., 2015). As polyene structure of lycopene makes it very sensitive in food processing (Wang, 2013; Silva et al., 2012), vulnerable to heat, light and pro-oxidants, the main change in its structure is the degradation and isomerization (Ines et al., 2013). Thus, it is meaningful to explore innovative technology in lycopene isomerization.

Cold sterilization technology has recently received greater attention, which not only can kill microorganisms in foods but also can maximally keep the color, flavor and nutritional ingredients of foods. Among them, there is no limitation of the size (Kumar and Sabhapathi, 2014) and geometry of the products (Kwon et al., 2010). Ultra-High Pressure technology (UHP) is a physical cold sterilization method. UHP can minimize damage to food ingredients (Shin et al., 2010; Park et al., 2012; Cho et al., 2013) and meet the high quality (Ferragut et al., 2015) and microbiological safety requirements of food under non-thermal conditions (Yan et al., 2015). Studies have shown that the ultra-high pressure treatment of carotenoids and lycopene can not only play a bactericidal role, but also make the whole trans carotenoids at 9, 13 and 15 sites with low energy to undergo partial cis isomerization or degradation to generate small molecular fragments, which can promote the conversion of lycopene into cis isomerization and bioavailability of active substances. However, there are no studies on the effect of UHP processing on isomerization of lycopene.

Hence, the aims of study were to give insight into the effect of UHP processing on isomerization of lycopene. In this study, UHP is used to treat lycopene, then HPLC is utilized to separate and identify the components of lycopene. Furthermore, antioxidant capability of the lycopene treated with UHP is evaluated.

\section{Materials and Methods}

\section{Material and Reagents}

Ingredients: Lycopene oleoresin (lycopene content 6\%, Xinjiang Tecom Biotechnology Co., Ltd., Xinjiang China); Reagents: Lycopene standards (97\% purity lycopene, Shanghai to Granville Chemical Co., Ltd., Shanghai China); hexane (AR, Tianjin Davydenko Davydenko chemical reagent Co., Tianjin China); acetonitrile, methanol, methyl tert-butyl ether, triethylamine (HPLC grade American sigma company, American) phenanthroline (AR, Shenyang reagent company, Shenyang China); 2-diphenyl-1-acid bitterness phenylhydrazine (DPPH·) (AR, Tianjin chemical reagent factory, Tianjin China).

\section{UHP Treatment of Lycopene Sample}

$2.5 \mathrm{mg}$ lycopene oleoresin was dissolved in $50 \mathrm{~mL}$ of $\mathrm{n}$-hexane, configurated concentration at $50 \mu \mathrm{g} / \mathrm{mL}$, sealed with double polyethylene plastic bags and packaged with vacuum. In order to study the effect of UHP on isomerization and antioxidation of lycopene, high pressure treatment were carried out at different degrees (400 Mpa, $500 \mathrm{Mpa}$ ), time ( $8 \mathrm{~min}, 10 \mathrm{~min}$ ) and low temperature (room temperature, $50^{\circ} \mathrm{C}$ ) while the specific operation followed to device instructions.

\section{$U v$-vis Spectra Identification of Lycopene Structure}

UV-visible spectrophotometer was used to scan the liquid of lycopene samples treated with UHP, with UHP untreated lycopene as control group. The differences between scanning spectra of the two samples were compared to determine whether there was the cislycopene after UHP treatment.

Lycopene isomers could be analyzed and identified according to retention time and spectral characteristics of high performance liquid chromatogram. According to preliminary research, cis-lycopene showed absorption peaks at 472 and $362 \mathrm{~nm}$ as well in the HPLC diagram. The ratio of this peak absorbance and the main peak absorbance were called $Q$ value, which is Acis/Atrans. At the same time, the maximum absorption wavelength $(\lambda \max )$ of main absorption peak of the cis-lycopene isomer moved to short-wavelength, "blue shift", direction 5-10 nm, compared to all-trans and the molar absorption coefficient decreased. Therefore, lycopene samples could be identified according to preliminary research.

\section{Isolation and Identification of Different Isomers of Lycopene by HPLC}

Different isomers of lycopene were isolated and identified by HPLC (Honda et al., 2017). Column: C30YMC CarotenoidS-5 $(4.6 \times 250 \mathrm{~mm})$; mobile phase A: Mixture of acetonitrile and methanol (acetonitrile: Methanol volume ratio was 3:1); mobile phase B: MTBE (methyl tert-butyl ether) was added. Triethylamine $(0.05 \%)$ was added to mobile phase A and B, respectively, eluted with gradient concentrations. Mobile phase B: The $8 \mathrm{~min}, 0 \sim 55 \%$ (V/V); 8 30 min: Maintaining 55\% (V/V), flow rate was $1 \mathrm{~mL} / \mathrm{min}$ (Li and Hui, 2010).

The content of cis and trans-isomers in the sample were determined according to the retention time and spectral characteristics of the separated components under the HPLC analysis conditions in terms of injection volume $10 \mu \mathrm{L}$, detection wavelength $471 \mathrm{~nm}$.

\section{Lycopene Standard Curve}

$10.02 \mathrm{mg}$ lycopene standard (HPLC $\geq 97 \%$ ) was accurately weighed, dissolved with $5 \mathrm{~mL}$ dichloromethane. Then $n$-hexane was used to set the volume to $50 \mathrm{~mL}$ to formulate $200 \mu \mathrm{g} / \mathrm{mL} \mathrm{n}$-hexane solution of lycopene and then this solution was diluted to lycopene standard solution series whose lycopene concentration was $20,40,60,80,100 \mu \mathrm{g} / \mathrm{mL}$, respectively. The area of peak of each solution was measured by HPLC, concentration of the lycopene 
standard solution was taken on the horizontal axis and peak area on the vertical axis, lycopene standard curve was plotted (Wang, 2013) and linear equation $\mathrm{Y}=$ $81.71 \times-11.51(\mathrm{R} 2=0.9639)$ was obtained.

\section{Antioxidant Capability Assay}

$\mathrm{N}$-hexane was used as solvent to prepare sample liquor whose concentration is $1 \mathrm{mg} / \mathrm{mL}$; the preparation of ascorbic acid liquor was consistent with the sample.

\section{Total Antioxidant Capability Assay}

The operation method was carried out according to the instructions of total antioxidant capacity assay (T-AOC):

Total antioxidation capability $(U / \mathrm{mg})$

$$
=(O D U-O D C) / 0.01 \div 30 \times N \div \text { Cprot }
$$

where, $O D U$ was the absorption value of sample; $O D C$ was the absoption value of control; $N$ represented the diluting times of system (which was to say, the ratio of total volume of reaction and sample volume); Cprot was the concentration of the sample to be measured.

Based on preliminary experiment, such us the linear relationship of standard $\mathrm{V}_{\mathrm{C}}$, concentration of the sample was set to $10,20,50,100,200,300 \mu \mathrm{g} / \mathrm{mL}$, respectively, concentration of the ascorbic acid was consist with sample.

\section{Hydroxyl Radical (• OH) Assay}

Preparation of the sample: The samples were weighed accurately, dissolved in distilled water and then n-hexane was added and used with ultrasonic extract 10 min to make lycopene transferred to the organic phase. It was repeated several times until the water layer became colorless. Organic phases were combined and dried by anhydrous sodium sulfate. Set the volume of organic phase to $50 \mathrm{~mL}$ and then diluted to formulate test solution whose concentration was $10,20,40,60,80,100$ $\mu \mathrm{g} / \mathrm{mL}$, respectively.

Phenanthroline - $\mathrm{Fe} 2^{+}$method was used. Firstly, $4 \mathrm{~mL}$ phosphate buffer ( $\mathrm{pH}$ 7.4) were added to a test tube and mixed with $1.5 \mathrm{~mL}$ of $5 \mathrm{mmol} / \mathrm{L} \mathrm{K} \mathrm{K}_{3} \mathrm{Fe}(\mathrm{CN})_{6}$ solution and blended thoroughly. Secondly, $1 \mathrm{~mL} 7.5 \mathrm{mmol} / \mathrm{L}$ ferrous sulfate solution was added, mixed immediately. Thirdly, $1 \mathrm{~mL}$ of sample was added, mixed immediately, then 1.5
$\mathrm{mL}$-hexane was added to supplement the volume. Lastly, $1 \mathrm{~mL} 0.1 \%$ hydrogen peroxide solution was added, mixed gently and then incubated for $1 \mathrm{~h}$ at $37^{\circ} \mathrm{C}$. The absorbance of the final solutions was measured at 536 $\mathrm{nm}$ with a UV/VIS spectrophotometer after holding them at $37^{\circ} \mathrm{C}$ for $1 \mathrm{~h}$.

\section{DPPH・Radical Assay}

$200 \mu \mathrm{g} / \mathrm{mL}$ lycopene liquor was diluted to the concentration $10,20,40,60,80,100 \mu \mathrm{g} / \mathrm{mL}$, respectively. $2 \mathrm{~mL}$ sample of each concentration was taken, $2 \mathrm{~mL}$ $2 \times 10-4 \mathrm{~mol} / \mathrm{L}$ DPPH.ethanol solution was added, vibrated, left in the dark for $30 \mathrm{~min}$ as a sample group; $\mathrm{DPPH}$-solution was replaced by an equal volume of nhexane in blank group; lycopene solution was replaced by an equal volume of ethanol in control group; an equal volume of a mixture of ethanol and n-hexane was taken as a blank zero. $A_{i}, A_{j}$ and $\mathrm{A} 0$ were measured at $517 \mathrm{~nm}$ and VC was taken as a positive control (Xu and Hang, 1999):

$D P P H \cdot$ scavenging ratio $\%=\left[1-\left(A_{i}-A_{j}\right) / A_{0}\right] \times 100$,

Where: $A_{i}$-sample group absorbance; $\mathrm{A}_{\mathrm{j}}$-blank group absorbance; $A_{0}$-control absorbance values.

\section{Statistical Analysis}

Excel 2007, GraphPad Prism 5.01, Origin 8.5, SPSS19.0 were carried out to analyze experimental data by ANOVA and process the graphics. Significance analysis was performed by using T-test, $\mathrm{P}<0.05$ was considered statistically significance while $\mathrm{P}<0.01$ indicated a significant difference.

\section{Results and Discussion}

\section{Effects of UHP Processing Conditions on Lycopene Isomerization}

High pressure treatment carried out at different pressures $(400,500 \mathrm{Mpa})$, time $(8,10 \mathrm{~min})$ and temperature (Room temperature, $50^{\circ} \mathrm{C}$ ) and degree of cis-isomerization of lycopene and content of lycopene were measured. The results were shown in Table 1.

Table 1: Effects of UHP processing conditions on lycopene isomerization

\begin{tabular}{lll}
\hline Sample & Cis-lycopene rate $(\%)$ & Content of lycopene $(\mathrm{mg} / \mathrm{g})$ \\
\hline Untreated sample & 13.25 & 52.37 \\
$400 \mathrm{Mpa}, 10 \mathrm{~min}$, Room temperature & 32.78 & 83.95 \\
$400 \mathrm{Mpa}, 10 \mathrm{~min}, 50^{\circ} \mathrm{C}$ & 34.76 & 87.94 \\
$400 \mathrm{Mpa}, 8 \mathrm{~min}, 50^{\circ} \mathrm{C}$ & 31.65 & 82.82 \\
$500 \mathrm{Mpa}, 10 \mathrm{~min}, 50^{\circ} \mathrm{C}$ & 45.69 & 103.24 \\
$500 \mathrm{Mpa}, 10 \mathrm{~min}, \mathrm{Room}$ temperature & 43.23 & 98.79 \\
$500 \mathrm{Mpa}, 8 \mathrm{~min}, 50^{\circ} \mathrm{C}$ & 39.51 & 97.67
\end{tabular}


As shown in Table 1, the proportion of cis-lycopene and content of lycopene under the condition of $500 \mathrm{Mpa}$, $10 \mathrm{~min}, 50^{\circ} \mathrm{C}$ was the highest, the second was under that of $10 \mathrm{~min}$, room temperature and the lowest was under that of $8 \mathrm{~min}, 50^{\circ} \mathrm{C}$ while under $400 \mathrm{Mpa}$ the results was similar to $500 \mathrm{Mpa}$, which may be because that high pressure treament caused the collide of the Van Der Waals forces between lycopene crystal molecules and $\pi$ electron orbital fracture, resulted in isomerization of lycopene and increased the proportion of cis-isomer (Jenny et al., 2001; Huo et al., 2011).

\section{Identification of Lycopene Isomers by UV-Vis}

By comparing the full wavelength scan of lycopene sample before and after UHP treatment, the paper found that the full wavelength scans of lycopene had been obviously changed after UHP treatment, appeared cis-peak at $362 \mathrm{~nm}$ as it had been reported in the literature (Xu and Hang, 1999), Fig. 1. There were three maximum absorption wavelength of lycopene at 447 , $471,503 \mathrm{~nm}$ respectively in the two full wavelength scans, but the full wavelength scans of lycopene after UHP treatment had a change: There was a characteristic absorption peak at $361 \mathrm{~nm}$, due to the transfer of trans-isomers into cis-isomer, thus it can be concluded that UHP could change lycopene structure.

Separation and Identification of Lycopene cisIsomer by HPLC

The lycopene oleoresin treated with UHP showed 5 more peaks than the standard lycopene (Fig. 2) and then UV-Vis spectra of the 5 peaks mentioned above were obtained after separated and preliminarily identified (Fig. 3). Moreover, the component Rt = 19.082 corresponding to was basically confirmed as

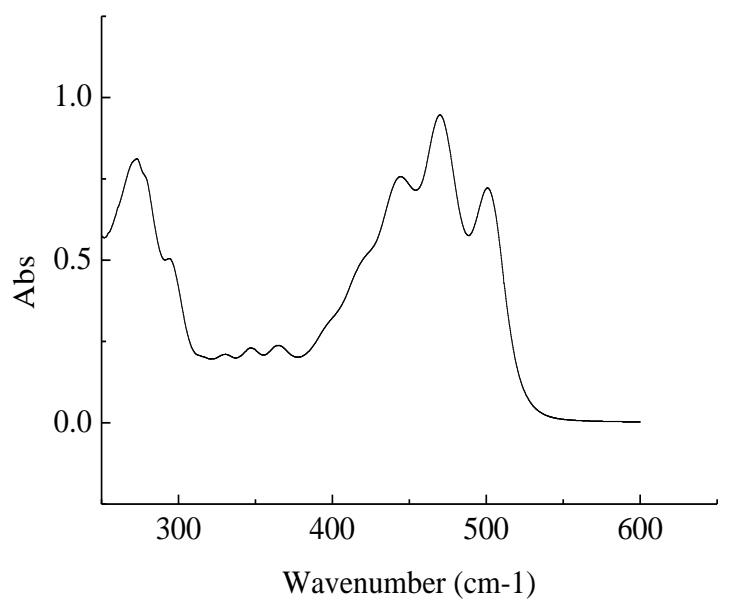

(a) all-trans lycopene due to its response time was consistent to that of all-trans lycopene while the peak pattern in UV-Vis spectra of the other components four peaks $(\mathrm{Rt}=13.307,9.790,8.123,7.207 \mathrm{~min})$ corresponding were similar to that of the all-trans lycopene in the 440-500 $\mathrm{nm}$ wavelength range, whose peak showed a purple shift of 3-10 nm and a strong absorption peak appeared at $361 \mathrm{~nm}$ which was consistent with UV-Vis spectra of cis-lycopene. Taken together, the 5 peaks mentioned above were the same type of substance and lycopene isomers. Furthermore, we preliminarily inferred that these four components $(\mathrm{Rt}=13.307,9.790,8.123,7.207 \mathrm{~min})$ were identified as the four kinds of cis-isomers of lycopene and could be separated for further study.

\section{Antioxidant Activity}

\section{Total Antioxidant Capability}

As what can be seen in Fig. 4: Total antioxidant activity of ascorbic acid, lycopene oleoresin, UHP lycopene oleoresin were measured and found. Total antioxidant capacity of these three materials were showed in the concentration dependent manner and good linear relationship ( $\mathrm{R}=0.9939,0.9618,0.9300$, respectively.). Within 10-300 $\mu \mathrm{g} / \mathrm{mL}$ concentration range, total antioxidant capacities of these three materials were: UHP lycopene oleoresin>lycopene oleoresin>ascorbic acid. There was no significant difference $(P>0.05)$ between the total antioxidant capacity of UHP lycopene oleoresin and that of lycopene oleoresin, but there was significantly difference $(P<0.01)$ between the total antioxidant capacity of UHP lycopene oleoresin and that of ascorbic acid, while that of ascorbic acid and lycopene oleoresin were significantly different $(P<0.05)$.

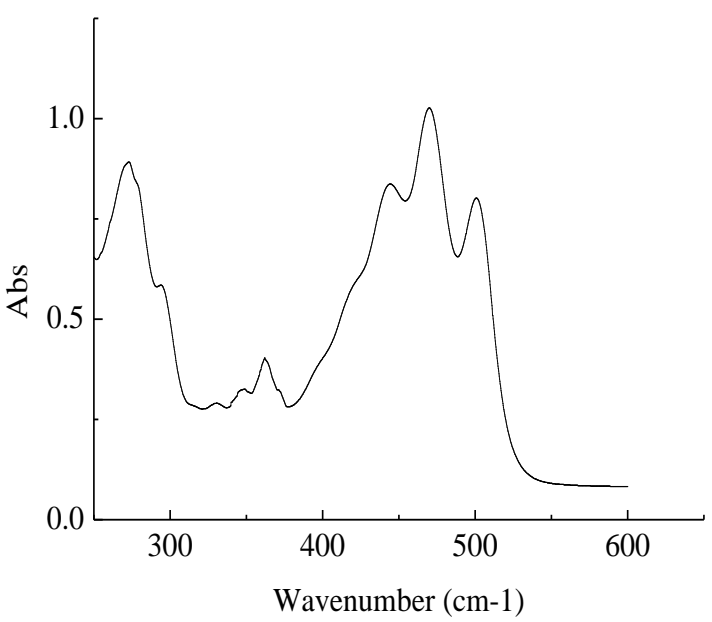

(b)

Fig. 1: The full wavelength scans of lycopene; (a) Before UHP; (b) After UHP 
Siqun Jing et al. / American Journal of Biochemistry and Biotechnology 2020, 16 (2): 270.279 DOI: 10.3844/ajbbsp.2020.270.279

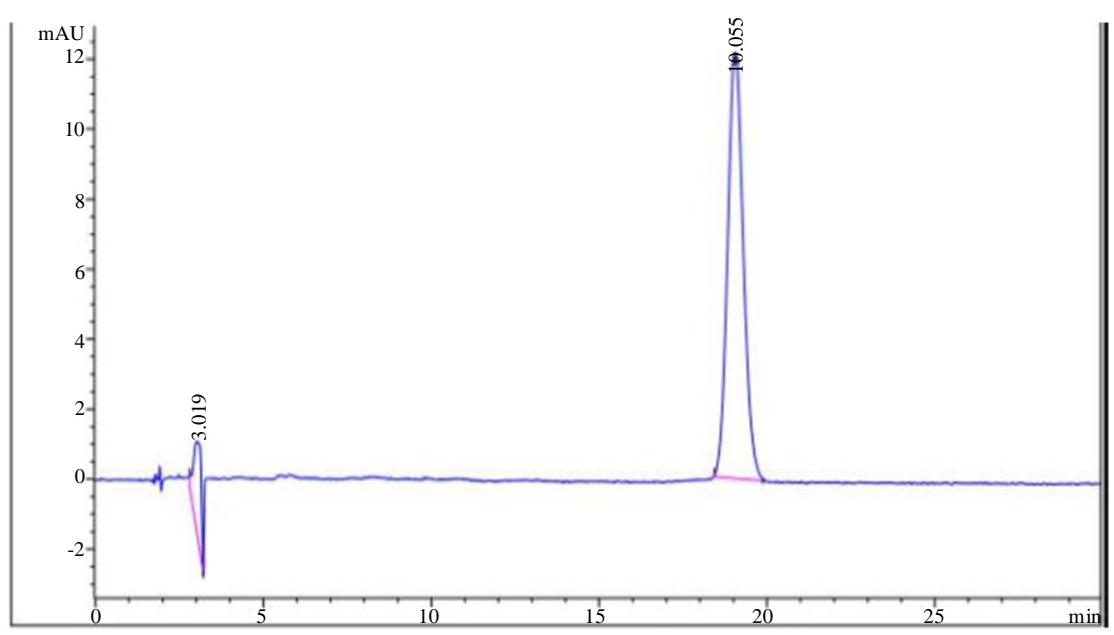

(a)

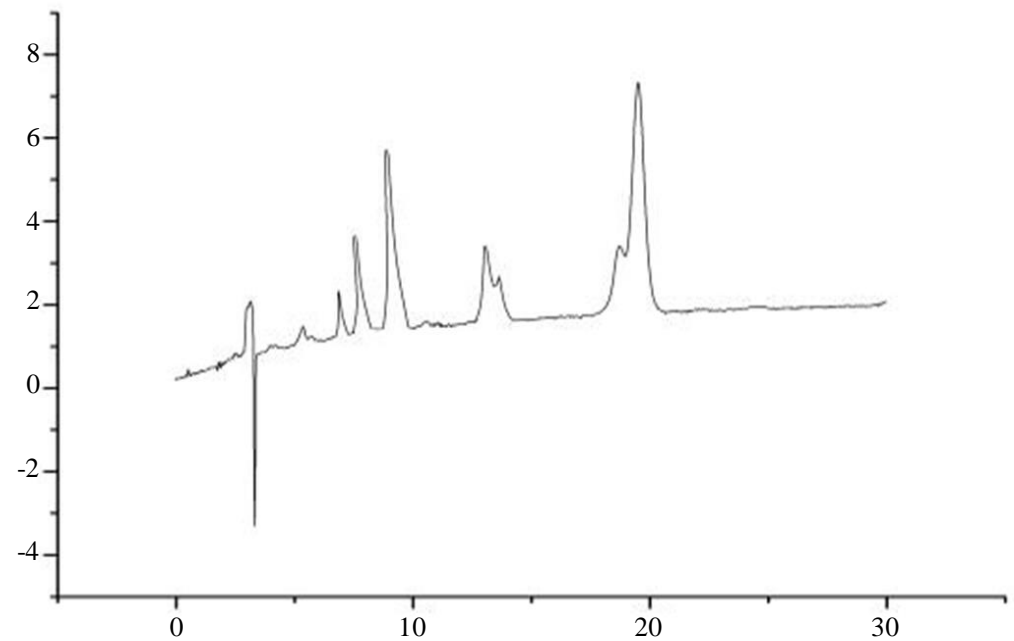

(b)

Fig. 2: HPLC spectrum; (a) HPLC spectrum of lycopene standard; (b) HPLC spectrum of lycopene oleoresin after UHP Note: $\mathrm{X}$ for retention time (min), $\mathrm{Y}$ for peak high (mAU)

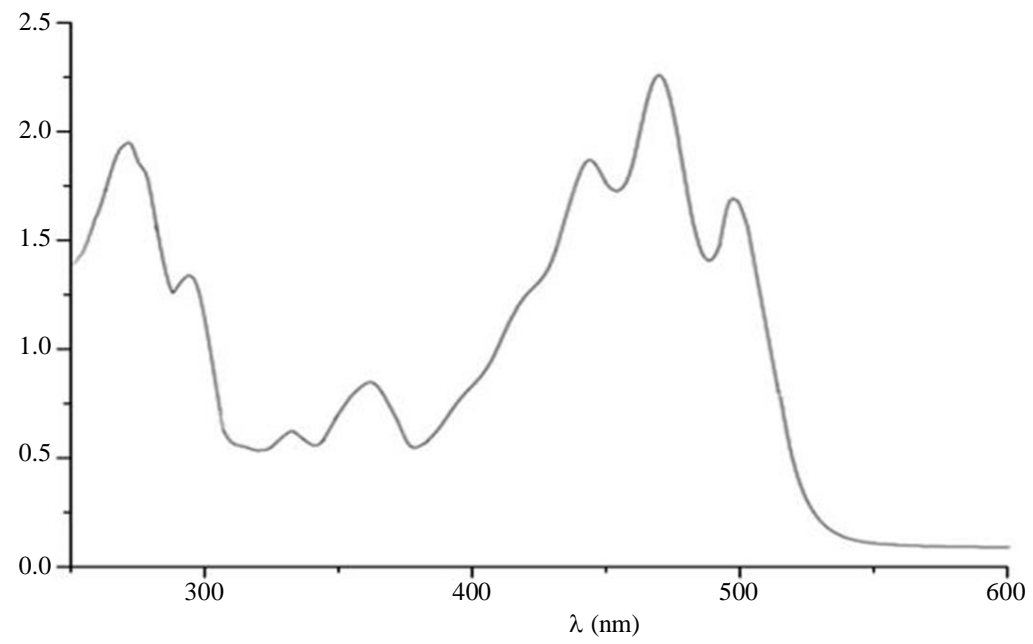

(a) 
Siqun Jing et al. / American Journal of Biochemistry and Biotechnology 2020, 16 (2): 270.279 DOI: 10.3844/ajbbsp.2020.270.279

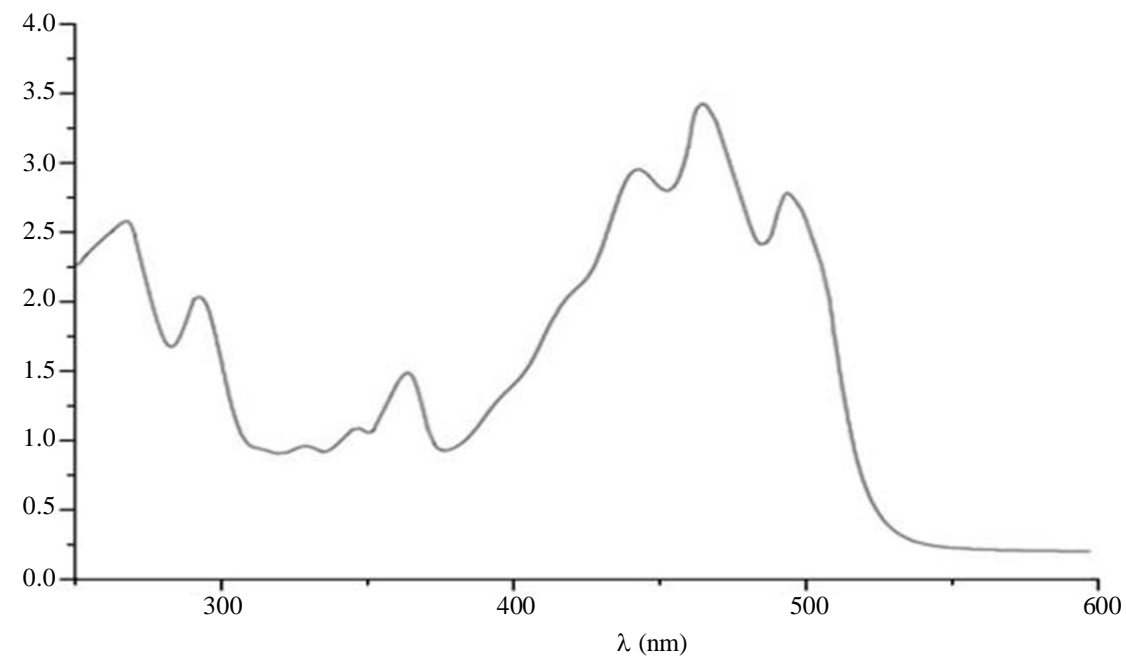

(b)

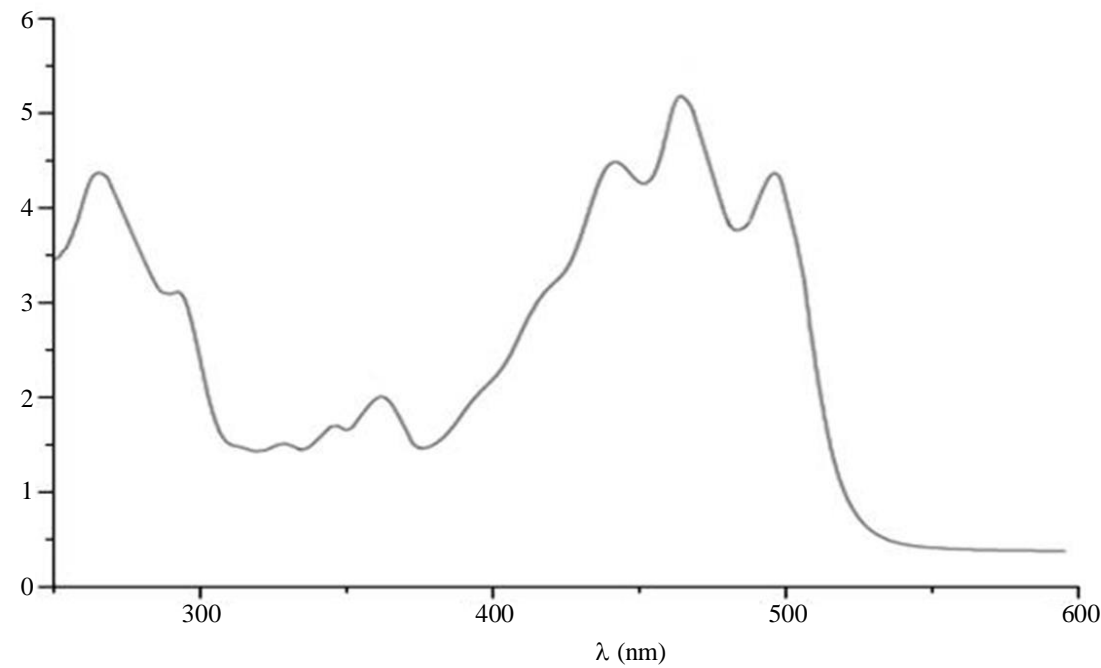

(c)

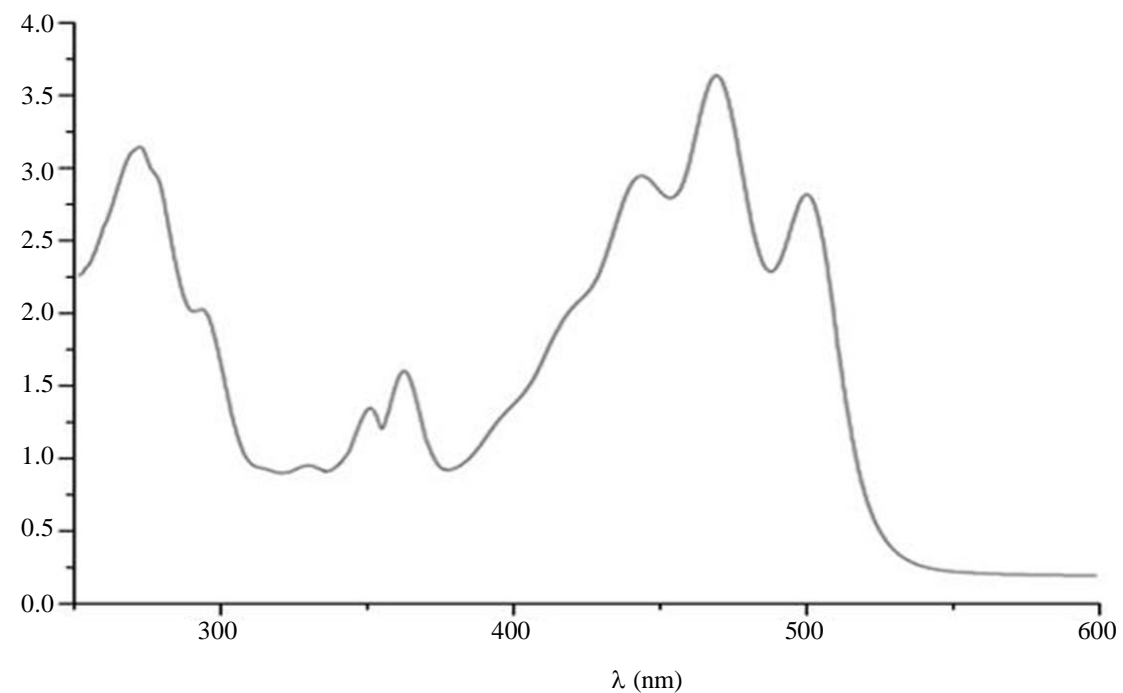

(d) 


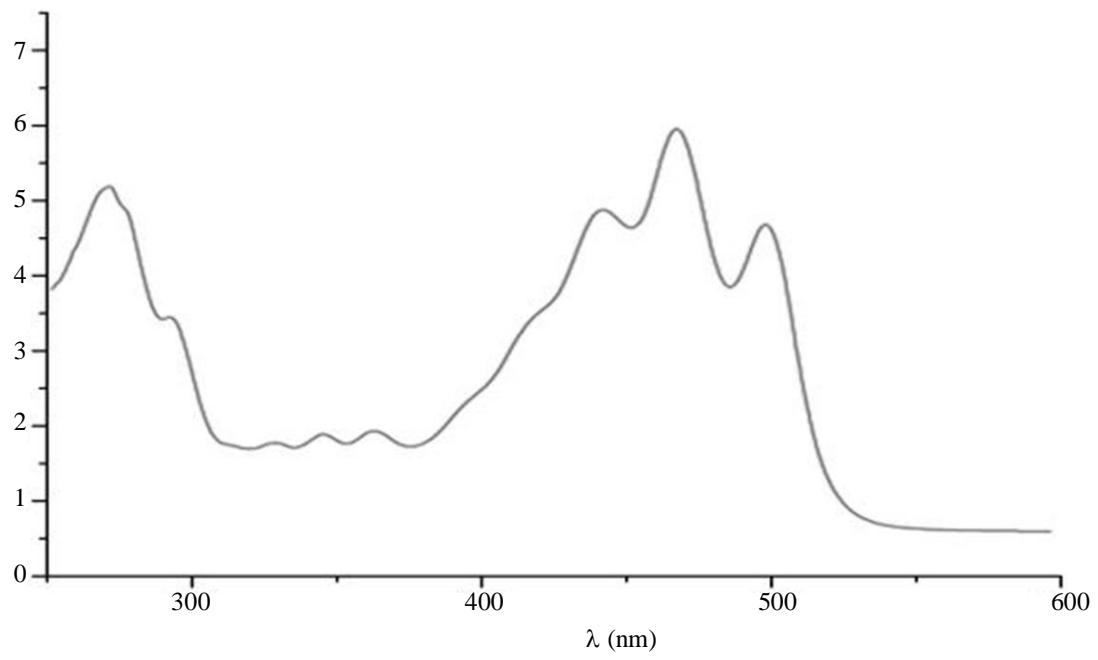

(e)

Fig. 3: The full wavelength scans of each HPLC peaks after UHP; (a) Peak 1; (b) Peak 2; (c) Peak 3; (d) Peak 4; (e) Peak 5 Note: $\mathrm{X}$ for $\lambda(\mathrm{nm}), \mathrm{Y}$ for peak high (mAU)

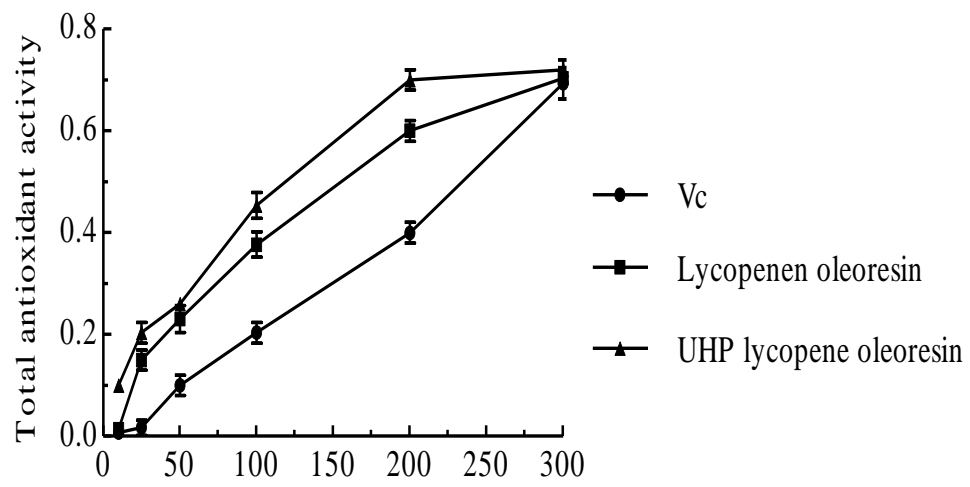

Concentration $(\mu \mathrm{g} / \mathrm{mL})$

Fig. 4: Total antioxidant capacity of lycopene product

\section{Hydroxyl Radical.OH Assay}

Hydroxyl radical, produced by the body during the metabolism process, is one of radicals which have strong toxicity to organisms. It contributes to the oxidation of sugars, amino acids, nucleic acids and other substances in body's tissues which results in various diseases and accelerate the aging of the body. Ascorbic acid, the samples of lycopene oleoresin before or after UHP treatment all had a significant scavenging effect on the hydroxyl radicals and showed a certain dose-dependent relationship (Fig. 5) with a good linear relationship $(\mathrm{R}=$ $0.9726,0.9412,0.9340$ respectively). $\mathrm{IC}_{50}$ value of ascorbic acid, lycopene oleoresin, UHP lycopene oleoresin were 45.6298, 40.2601, $24.0639 \mu \mathrm{g} / \mathrm{mL}$, respectively. Therefore, scavenging hydroxyl radical capabilities of these three substances were: UHP lycopene oleoresin> lycopene oleoresin> ascorbic acid. There were significant differences $(P<0.01)$ between the scavenging hydroxyl radical capability of the UHP lycopene oleoresin and that of lycopene oleoresin, but no significant difference $(P>0.05)$ with ascorbic acid and there was no significant difference $(P>0.05)$ between ascorbic acid and lycopene oleoresin.

\section{DPPH·radical Assay}

As it can be seen from Fig. 6 all of the ascorbic acid, lycopene oleoresin, UHP lycopene oleoresin had a strong DPPH.radical scavenging capability. All three of them showed a certain dose-dependent relationship over a range of $20-100 \mu \mathrm{g} / \mathrm{mL}$ with a good linear relationship $\left(\mathrm{R}=0.9695,0.9476,0.8990\right.$, respectively). $\mathrm{IC}_{50}$ value of ascorbic acid, lycopene oleoresin, UHP lycopene oleoresin was $47.3698,52.0821,39.6950 \mu \mathrm{g} / \mathrm{mL}$, respectively. 


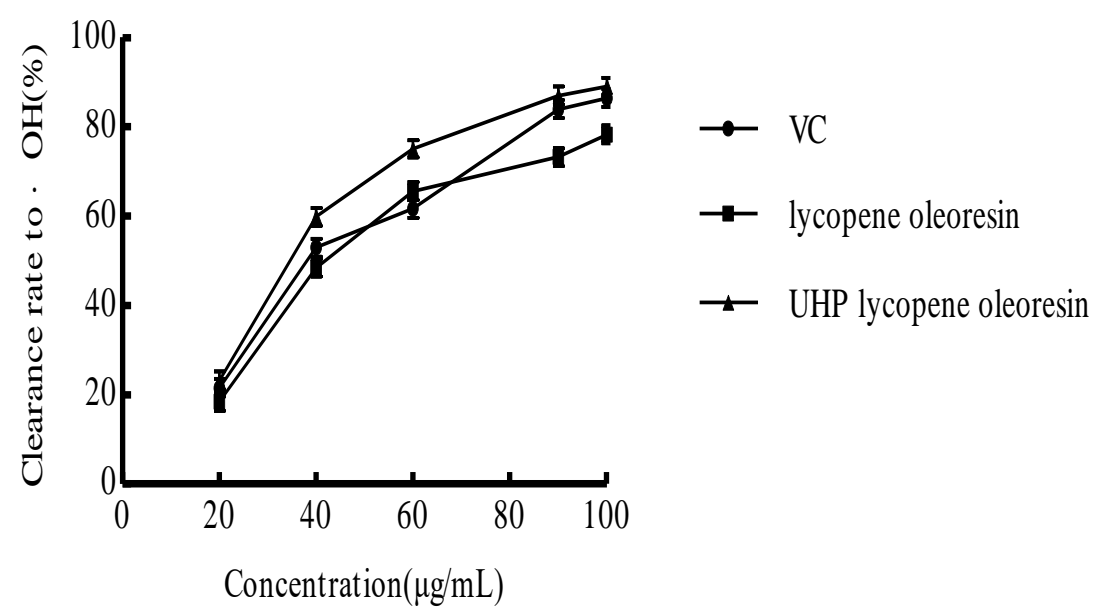

Fig. 5: Ability of lycopene products clear hydroxyl radical $(\cdot \mathrm{OH})$

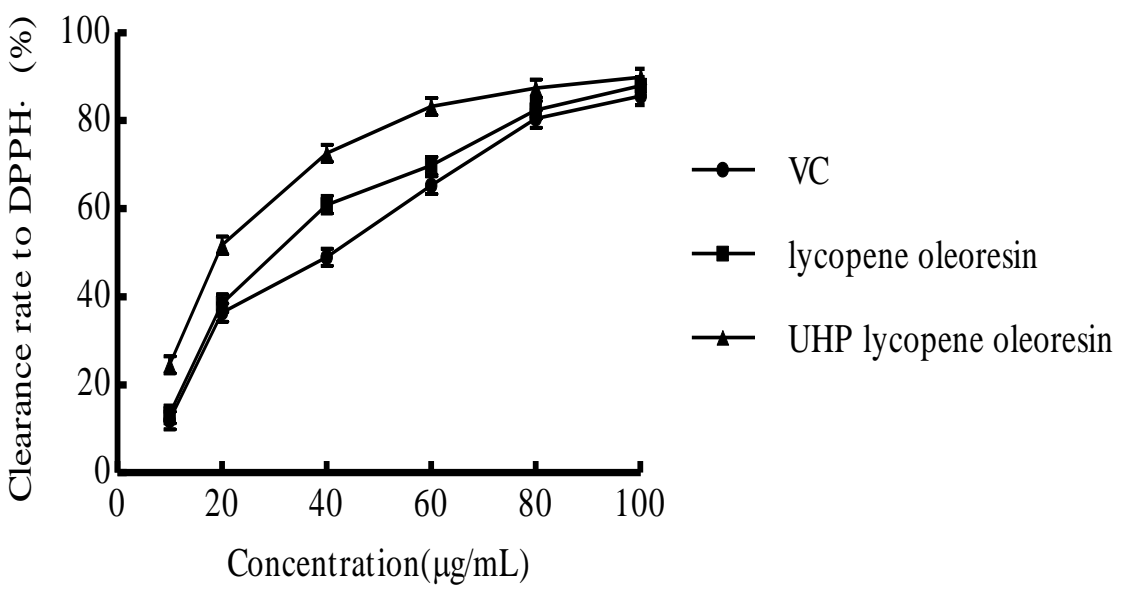

Fig. 6: Ability of lycopene products clear diphenyl picryl hydrazinyl radical (.DPPH)

Thus, the capability of these three substances to scavenge DPPH.radical was: UHP lycopene oleoresin>ascorbic acid>lycopene oleoresin. There were significant difference $\quad(P<0.01) \quad$ between the scavenging DPPH -capability of UHP lycopene oleoresin and that of ascorbic acid and lycopene oleoresin, while there was no significant difference $(P>0.05)$ between that of ascorbic acid and lycopene oleoresin.

\section{Conclusion}

In this study, we found that pretreatment with UHP could not only effificiently improve the proportion and content of cis-isomer of lycopene in oleoresin but also strengthen the antioxidant activity of lycopene in vitro. Therefore, we can conclude that UHP treatment was a promising method for converting lycopene into cis-trans isomerism with higher antioxidant activity. However, further work will be needed to raise the cis-isomer proportion of lycopene to more than $50 \%$ by UHP.

\section{Acknowledgement}

This work was financially supported by the National Science Foundation of China (No.31660462) and Professor Research Start-Up Fee of Shaoguan University (No. 433-99000611).

\section{Author's Contributions}

Siqun Jing: She designed the research plan and organized the study.

Hui Shi: She participated in all experiments, coordinated the data-analysis and contributed to the writing of the manuscript.

Saisai Wang: She participated to collect the materialsrelated to the experiment. 
Zainixi Lasheng: She contributed to the writing of the manuscript.

\section{Conflict of Interest}

The authors declare that they have no competing interests. The corresponding author affirms that all of the authors have read and approved the manuscript.

\section{Ethics}

This article is original and contains unpublished material. The corresponding author confirms that all of the other authors have read and approved the manuscript and no ethical issues involved.

\section{References}

Armoza, A., Y. Haim and A. Basiri, 2012. Tomato extract and the carotenoids lycopene and lutein improve endothelial function and attenuate inflammatory NF-B signaling in endothelial cells. J. Hypertens, 52: 41-47. DOI: 10.1097/HJH.0b013e32835c1d01

Cho, A., S.H. Choi, H.W. Choi, H.S. Kim and W. Kim et al., 2013. Characterization of cationic dextrin prepared by Ultra High Pressure (UHP)assisted cationization reaction. Carbohydr Polym., 97: 130-137. DOI: 10.1016/j.carbpol.2013.04.038

Cooperstone, J., D. Francis and S. Schwartz, 2016. Thermal processing differentially affects lycopene and other carotenoids in cis-lycopene containing, tangerine tomatoes. Food Chem., 210: 466-472. DOI: 10.1016/j.foodchem.2016.04.078

Ferragut, V., M. Hemandezherrero, M.T. Veciananogues, M. Borras-Suarez and J. González-Linares et al., 2015. Ultra-High-Pressure Homogenization (UHPH) system for producing high-quality vegetable-based beverages: Physicochemical, microbiological, nutritional and toxicological characteristics. J. Sci. Food Agric., 95: 953-961. DOI: 10.1002/jsfa.6769

Honda, M., T. Kudo, T. Kuwa, T. Higashiura and T. Fukaya et al., 2017. Isolation and spectral characterization of thermally generated multi-\r, Z $\backslash r$, -isomers of lycopene and the theoretically preferred pathway to di- $\backslash$ r, Z $\mathrm{rr}$, -isomers. Biosci. Biotechnol. Biochem., 81: 365-371.

DOI: 10.1080/09168451.2016.1249454

Huo, M.M., W.L. Liu, Z.R. Zheng, W. Zhang and A.H. Li et al., 2011. Effect of end groups on the raman spectra of lycopene and $\beta$-carotene under high pressure. Molecules, 16: 1973-1980.

DOI: 10.3390/molecules16031973
Ines, J.P., L. Colle, S. Lemmens, A.M. Van Loey and M.E. Hendrickx, 2013. Modeling lycopene degradation and isomerization in the presence of lipids. Food Bioprocess Technol., 6: 909-918. DOI: $10.1007 / \mathrm{s} 11947-011-0714-4$

Jenny, C.Y., Yeung, A. Gregory, L.L. Torday and J.G. Papp, 2001. Cationic intermediates in trans-to cisisomerization reactions of allylic systems. An exploratory ab initio study. J. Molecular Structure (Theochem), 546: 143-162. DOI: $10.1016 /$ S0166-1280(01)00441-9

Kumar, R. and S.N. Sabhapathi, 2014. Non thermal food processing technologies and microbiological safety. Proceedings of the 55th Conference of Association of Microbiologist of India, (AMI' 14).

Kwon, D.Y., J.S. Jang, J.E. Lee, Y.S. Kim and D.H. Shin et al., 2010. The is flavonoid aglyconerich fractions of Chungkookjang, fermented unsalted soybeans, enhance insulin signaling and peroxisome proliferator-activated receptor-yactivity in vitro. Bio-Factors, 26: 245-258.

DOI: $10.1002 /$ biof.5520260403

Li, J. and B.L. Hui, 2010. Analysis of lycopene content and geometric isomer composition in autumn olive fruit. Sci. Technol. Food Industry, 10: 64-65.

Li, X.N., J. Lin, J. Xia, L. Qin and S.Y. Zhu et al., 2017. Lycopene mitigates atrazine-induced cardiac inflammation via blocking the $\mathrm{nf}-\kappa \mathrm{b}$ pathway and no production. J. Funct. Foods, 29: 208-216.

DOI: $10.1016 /$ j.jff.2016.12.029

Murakami, K., M. Honda, R. Takemura, T. Fukaya and M. Kubota et al., 2017. The thermal zisomerization-induced change in solubility and physical properties of (all-e)-lycopene. Biochem. Biophys. Res. Commun., 19: 743-748.

DOI: 10.1016/j.bbrc.2017.07.103

Park, K., E. Cho, M.J. In, D.C. Kim and H.J. Chae, 2012. Physicochemical properties and bioactivity of brown seaweed fucoidan prepared by ultra high pressureassisted enzyme treatment. Korean J. Chem. Eng., 29: 221-227. DOI: 10.1007/s11814-011-0165-7

Peng, S.J., J. Li, Y. Zhou, M. Tuo and X.X. Qin et al., 2017. In vitro effects and mechanisms of lycopene in mcf-7 human breast cancer cells. Genet. Molecular Res. Gmr, 16: 1-8. DOI: 10.4238/gmr16029434

Petyaev, I.M., P.Y. Dovgalevsky, N.E. Chalyk, V. Klochkov and N.H. Kyle, 2014. Reduction in blood pressure and serum lipids by lycosome formulation of dark chocolate and lycopene in prehypertension. Food Sci. Nutrit., 2: 744-750. DOI: 10.1002/fsn3.169

Phan-Thi, H. and Y. Wacha, 2014. Isomerization and increase in the antioxidant properties of lycopene from Momordica cochinchinensis (GAC) by moderate heat treatment with UV-Vis spectra as a marker. Food Chem., 156: 58-63.

DOI: 10.1016/j.foodchem.2014.01.040 
Saedisomelia, A., L.B.G. Wood and M.L. Garg, 2009. Lycopene enrichment of cultured airway epithelial cells decreases the inflammation induced by rhinovirus infection and lipopolysaccharide. J. Nutr. Biochem., 20: 577-585. DOI: 10.1016/j.jnutbio.2008.06.001

Silva, D., C. Favaro, G. Rocha and M. Thomazini, 2012. Microencapsulation of lycopene by gelati-pectin complex coacervation. J. Food Process. Preservat., 36: 185-190. DOI: $10.1111 / j .1745-4549.2011 .00575 . x$

Sy, C., O. Dangles, P. Borel and C. Caris-Veyrat, 2015. Interactions between carotenoids from marine bacteria and other micronutrients: Impact on stability and antioxidant activity. Marine Drugs, 13: 7020-7039. DOI: 10.3390/md13117020

Shin, J.S., S.C. Ahn, S.W. Choi, D.U. Lee and B.Y. Kim et al., 2010. Ultra High Pressure Extraction (UHPE) of ginsenosides from Korean Panax ginseng, powder. Food Sci. Biotechnol., 19: 743-748. DOI: 10.1007/s10068-010-0104-0
Takehara, M., T. Kuwa, Y. Inoue, C. Kitamura and M. Honda, 2015. Isolation and characterization of (15Z)-lycopene thermally generated from a natural source. Biochem. Biophys. Res. Commun., 467: 58-62. DOI: 10.1016/j.bbrc.2015.09.122

Wang, X.W., 2013. Study on the configuration-changing and stability of lycopene in the process of microemulsion preparation and storage. J. Food Sci. Biotechnol., 32: 22-29.

$\mathrm{Xu}$, S.H. and H. Hang, 1999. Study on antioxidant activity of wild plants by analytical method. J. Qingdao Univ., 12: 75-78.

Yan, H., D. Miao, F. Nan and X. XiuFang, 2015. Effect of ultra-high pressure technology on microbial and quality of meat products. J. Food Safety Quality, 6: 545-549. 